\title{
Can Methotrexate Prevent Knee Arthro- plasties in Patients with Rheumatoid Arthritis?
}

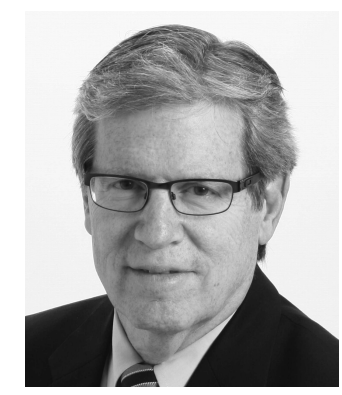

In this issue of The Journal a Japanese group reports an overall diminished incidence of total knee arthroplasty (TKA) in a group of patients with longstanding rheumatoid arthritis (RA) receiving methotrexate (MTX) plus tumor necrosis factor (TNF) inhibitor, versus another group receiving TNF inhibitor but not MTX $^{1}$.

If true, the findings are certainly of interest. Perhaps one of the first questions that might occur to a clinical rheumatologist is this: How many subjects may have had knee osteoarthritis (OA) leading to their surgical procedure and how many may have had knee disease secondary to RA, or some combination of both?

Patients were well matched for age and disease duration although fewer knee arthroplasties were performed in subjects receiving infliximab (IFX) versus those taking etanercept (ETN). Adalimumab was not included because it was apparently not prescribed in the Japanese population studied at the single center during the duration of the trial.

Because modifications of the Sharp scores typically used in radiographic studies of patients with RA predominantly report outcomes of small joints of the hands and wrists (because of the sheer predominance of these joints in the scoring system), a focus on large joints is uniquely interesting. However, this editorialist finds so many methodological challenges with the trial, that it is difficult to have a high degree of confidence in the authors' conclusion that MTX could indeed be protective for the outcome of TKA.

The authors were able to study only 68 patients retrospectively; moreover, they doubled their analyzable numbers by considering each knee separately. There is reason to question this approach because the biologic and biomechanical milieu is likely to be quite similar in both knees of an individual. It should also be noted that only 16 patients received a TNF inhibitor without MTX.

All the IFX patients received MTX, and most of the MTX-negative patients were taking ETN. While the authors report a median weekly dose of MTX of $8 \mathrm{mg}$ (range 6-10), the dose of IFX is not reported. In addition, $68 \%$ of the knees that were arthroplasty-free were from subjects taking corticosteroids, versus $41 \%$ that had a knee replacement. No information is provided regarding intraarticular steroids. Systemic corticosteroids can, of course, inhibit bony erosion and cartilage breakdown.

There are several other potential confounders or omissions which, if better defined, would have resulted in a higher degree of confidence in the authors' findings, including the absence of data on the prevalence of radiographic features of advanced OA at the time of arthroplasty, or actual surgical findings, which might have confirmed OA or RA. As noted, the numbers are also quite small.

Nevertheless it must be said that the authors deserve credit for seeking to examine the outcome of different interventions on large, weight-bearing joints in patients with RA.

Thinking purely scientifically, we cannot eliminate the possibility that there may indeed be an effect of MTX on large joint deterioration. The authors suggest that a randomized controlled trial will need to be performed, but it is highly unlikely that such a trial could ever be conducted over the time course required for the outcomes of interest to become apparent. That is, it would be unethical to sustain a monotherapy group that is doing poorly in a head-to-head trial. Indeed, observational or administrative claims data are much more likely to provide further evidence for, or against, the authors' hypothesis.

A few other comments are relevant. As the authors point out, the CONCERTO trial ${ }^{2}$ did demonstrate that even low doses of MTX can be useful when used with a TNF inhibitor. And we know that both the TEMPO ${ }^{3}$ and PREMIER trials ${ }^{4}$ clearly demonstrated that a combination of MTX and a TNF inhibitor is superior to either drug alone.

It is worth noting in this context that Dervieux and colleagues have demonstrated that MTX may inhibit antidrug antibodies to IFX, thus rendering this agent more

See Concomitant MTX protects against TKA, page 2255

Personal non-commercial use only. The Journal of Rheumatology Copyright @ 2015 . All rights reserved. 
efficacious ${ }^{5}$. IFX trough serum levels were higher in subjects who demonstrated higher MTX polyglutamate levels in this study, and these subjects exhibited the best response to treatment. It is quite possible, as seen in the CONCERTO trial, that even the lower doses of weekly MTX, as used in the Japanese population, are able to achieve these same results when used in combination with a TNF inhibitor.

More data will be needed on much larger numbers of patients to be able to assess the possible contribution of MTX to the avoidance of either total knee or hip arthroplasty. While the present study can be conceptualized as hypothesis generating, the effect of MTX on these outcomes in patients with RA remains quite uncertain. Perhaps observational data on much larger populations can begin to illuminate the possibility that MTX could deter, or slow, cartilage and/or bone deterioration that lead to the need for joint replacement.

JOEL M. KREMER, MD,

Pfaff Family Professor of Medicine, Albany Medical College, Director of Research,

Center for Rheumatology, 1367 Washington Ave., Albany, New York 12206, USA.

Address correspondence to Dr. J.M. Kremer;

E-mail: jkremer@joint-docs.com

\section{REFERENCES}

1. Asai S, Takahashi N, Funahashi K, Yoshioka Y, Takemoto T, Terabe $\mathrm{K}$, et al. Concomitant methotrexate protects against total knee arthroplasty in patients with rheumatoid arthritis treated with tumor necrosis factor inhibitors. J Rheumatol 2015;42:2255-60.

2. Burmester GR, Kivitz AJ, Kupper H, Arulmani U, Florentinus S, Goss SL, et al. Efficacy and safety of ascending methotrexate dose in combination with adalimumab: the randomized CONCERTO trial. Ann Rheum Dis 2015;74:1037-44.

3. Klareskog L, van der Heijde D, de Jager JP, Gough A, Kalden J, Malaise M, et al. Therapeutic effect of the combination of etanercept and methotrexate compared with each treatment alone in patients with rheumatoid arthritis: double-blind randomized controlled trial. Lancet 2004;363:675-81.

4. Breedveld FC, Weisman MH, Kavanaugh AF, Cohen SB, Pavelka $\mathrm{K}$, van Vollenhoven R, et al. The PREMIER study: A multicenter, randomized, double-blind clinical trial of combination therapy with adalimumab plus methotrexate versus methotrexate alone or adalimumab alone in patients with early, aggressive rheumatoid arthritis who had not had previous methotrexate treatment. Arthritis Rheum 2006;54:26-37.

5. Dervieux T, Weinblatt M, Kivitz A, Kremer JM. Methotrexate polyglutamation in relation to infliximab pharmacokinetics in rheumatoid arthritis. Ann Rheum Dis 2013;72:908-10.

J Rheumatol 2015;42:2217-8; doi:10.3899/jrheum.151104 